\title{
Rare four-leptonic B-decays with light leptons in the final state in the Standard Model
}

\author{
Anna Danilina ${ }^{1,2,3, \star}$ and Nikolai Nikitin ${ }^{1,2,3, \star \star}$ \\ ${ }^{1}$ M. V.Lomonosov Moscow State University, Physics Faculty \\ ${ }^{2}$ D. V.Skobeltsyn Institute of Nuclear Physics \\ ${ }^{3}$ Institute for Theoretical and Experimental Physics
}

\begin{abstract}
We present predictions for the four-leptonic decays of $B^{-}$-mesons with three light charged leptons and one neutrino in the final states. We obtain the branching ratio for $B^{-} \rightarrow \mu^{+} \mu^{-} e^{-} \bar{v}_{e}$ decay at the level $1.3 \times 10^{-7}$ taking into account the photon emission from the $u$ - and $b$ - valence quarks of $B^{-}$-meson and the Bremsstrahlung. The contribution of the virtual photon was calculated using the Vector Meson Dominance model (VMD). As a next step we present prediction for branching ratio of the decay $B^{-} \rightarrow \mu^{-} \mu^{+} \mu^{-} \bar{v}_{\mu}$ and the estimation for $\operatorname{Br}\left(B_{s} \rightarrow \mu^{+} \mu^{-} e^{+} e^{-}\right)$.
\end{abstract}

\section{Introduction}

The investigation of rare four leptonic decays of the $B$-mesons provides an important test of the Standard Model (SM) and its possible extensions in the high order of the perturbation theory. The branching ratios of these decays are sufficiently sensitive to the contribution of the "New Physics". Therefore it is very important to precisely predict different characteristics of the $B$-mesons decays. Four-lepton $B$-decays are investigated at the LHCb, CMS and ATLAS experiments at LHC. By now, only upper limits on the branching ratios for the decays $B_{d, s} \rightarrow \mu^{+} \mu^{-} \mu^{+} \mu^{-}$were obtained at the LHCb [1, 2].

\section{Total Hamiltonian for the decays $B^{-} \rightarrow \ell^{+} \ell^{-} \ell^{\prime-} \bar{v}_{\ell^{\prime}}$}

The total Hamiltonian for four-leptonic decays $B^{-} \rightarrow \ell^{+} \ell^{-} \ell^{\prime-} \bar{v}_{\ell^{\prime}}$ in the Standard Model is given by following expression:

$$
\mathcal{H}_{\text {tot }}(x)=\mathcal{H}_{u b}(x)+\mathcal{H}_{e m}(x)+\mathcal{H}_{V M D}(x) .
$$

The Hamiltonian relevant for the transition of interest $b \rightarrow u W^{-} \rightarrow u \ell^{-} \bar{v}_{\ell}$ has the form

$$
\mathcal{H}_{u b}(x)=\frac{G_{F}}{\sqrt{2}} V_{u b}\left(\bar{u}(x) \gamma^{\mu}\left(1-\gamma^{5}\right) b(x)\right)\left(\bar{\ell}(x) \gamma_{\mu}\left(1-\gamma^{5}\right) v_{\ell}(x)\right)+\text { h.c. }
$$

with $G_{F}$ the Fermi constant, $V_{u b}$ the CKM-matrix element, and $\gamma^{5}$ defined as $\gamma^{5}=i \gamma^{0} \gamma^{1} \gamma^{2} \gamma^{3}$.

\footnotetext{
$\star$ e-mail: anna.danilina@cern.ch

$\star \star$ e-mail: nnikit@mail.cern.ch
} 
The electromagnetic Hamiltonian is given by

$$
\mathcal{H}_{e m}(x)=e \sum_{f} Q_{f}\left(\bar{f}(x) \gamma^{\mu} f(x)\right) A_{\mu}(x),
$$

where $e=|e|>0$ is the elementary charge, $e^{2}=4 \pi \alpha_{e m}, \alpha_{e m} \approx 1 / 137$ the fine structure constant, $Q_{f}$ the charge of a fermion with flavour $f$ in units of elementary charge.

The Hamiltonian describing the Vector Meson Dominance model has the form

$$
\mathcal{H}_{V M D}(x)=\sum_{V} \frac{e M_{V}^{2}}{f_{V}} V^{\mu}(x) A_{\mu}(x),
$$

$M_{V}$ - is the mass of corresponding vector meson, $V^{\mu}(x)$ - is the vector meson field. The absolute value of the VMD-constant $f_{V}$ is obtained from

$$
\Gamma\left(V \rightarrow \ell^{+} \ell^{-}\right)=\frac{4 \pi}{3}\left(\frac{\alpha_{e m}}{\left|f_{V}\right|}\right)^{2} M_{V}
$$

It is assumed that VMD-constants $f_{V}$ for $\rho$ and $\omega$ mesons are real and positive.

\section{The $B^{-} \rightarrow \mu^{+} \mu^{-} e^{-} \bar{v}_{e}$ amplitude of the decay}

The amplitude of the decays $B^{-}(p) \rightarrow \ell^{+}\left(k_{1}\right) \ell^{-}\left(k_{2}\right) \bar{v}_{\ell^{\prime}}\left(k_{3}\right) \ell^{\prime-}\left(k_{4}\right)$ involves several different contributions. Firstly, it is the photon emission from the valence $u$-quark of the $B^{-}$-meson; the corresponding diagram is given in Fig. 1.

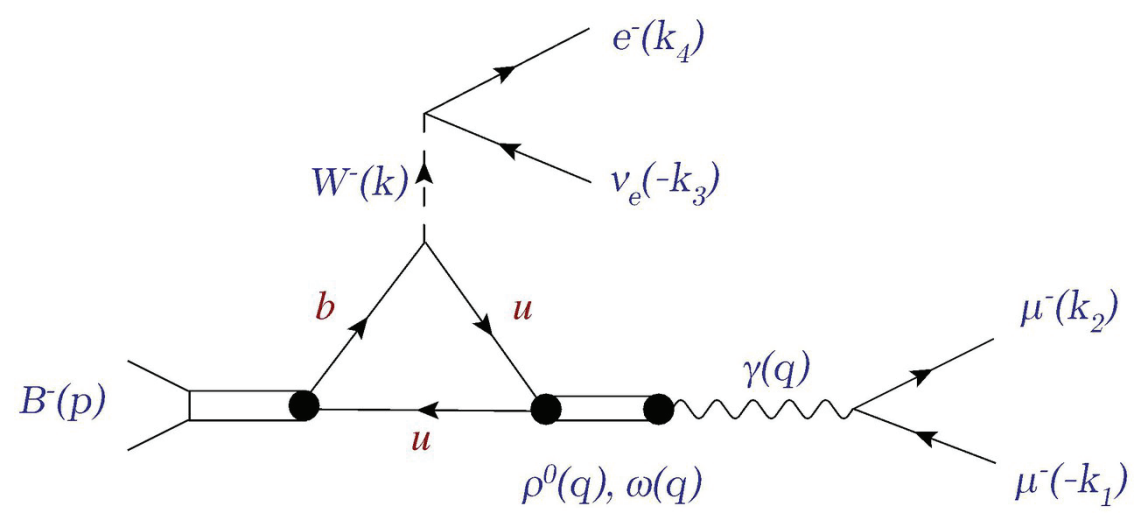

Figure 1. Emission of the virtual photon from $u$-quark in the VMD approach.

In this case we described the photon emissions via VMD includes the intermediate $\rho^{0}(770)$ and $\omega(782)$ mesons. The amplitude corresponding to the diagram of Fig. 1 can be parametrized as follows

$$
F^{(u)}\left(q^{2}, k^{2}\right)=\sum_{i} \frac{M_{2 i}^{2}}{q^{2}} \frac{M_{1}^{2}}{q^{2}-M_{2 i}^{2}+i \Gamma_{2 i} M_{2 i}} G^{(u)}\left(q^{2}, k^{2}, M_{2 i}^{2}\right),
$$

where $M_{2 i}$ and $\Gamma_{2 i}$ - are the mass and the full width of the intermediated vector meson that contains $u \bar{u}$-pair, $G^{(u)}\left(q^{2}, k^{2}, M_{2 i}^{2}\right)$ - the smooth function without poles, $q=k_{1}+k_{2}$ and $k=k_{3}+k_{4}$. 
The function $F^{(u)}\left(q^{2}, k^{2}\right)$ has the poles at the $q^{2}=M_{2 i}^{2}$.

The leftmost singularity in the $k^{2}$-variable lies at $k^{2}=M_{B^{*-}}^{2}$. This singularity was excluded from the kinematic region which was taken into account in the transition form factors $B \rightarrow \rho$ and $B \rightarrow \omega$ parameterization.

Before calculating the decay rate, it is useful to estimate the contribution of all diagrams. The contribution of the diagram Fig. 1 can be estimated roughly considering the conrtibution of $\rho^{0}(770)$ and $\omega(782)$ intermediate mesons:

$$
\begin{aligned}
\operatorname{Br}^{(V M D)}\left(B^{-}\right. & \left.\rightarrow \mu^{+} \mu^{-} e^{-} \bar{v}_{e}\right) \approx \\
& \approx \mid \sqrt{\operatorname{Br}\left(B^{-} \rightarrow \rho^{0} e^{-} \bar{v}_{e}\right) * \operatorname{Br}\left(\rho^{0} \rightarrow \mu^{+} \mu^{-}\right)}+ \\
& +\left.\sqrt{\operatorname{Br}\left(B^{-} \rightarrow \omega e^{-} \bar{v}_{e}\right) * \operatorname{Br}\left(\omega \rightarrow \mu^{+} \mu^{-}\right)}\right|^{2} \approx 0,4 \times 10^{-7}
\end{aligned}
$$

An alternative way to estimate of the contribution from the diagram Fig. 1 is to represent $B^{-} \rightarrow$ $\mu^{+} \mu^{-} e^{-} \bar{v}_{e}$ decay as a chain of processes $B^{-} \rightarrow \gamma^{*} e^{-} \bar{v}_{e} \rightarrow \mu^{+} \mu^{-} e^{-} \bar{v}_{e}$.

The branching ratio of $B^{-} \rightarrow e^{-} \bar{v}_{e}$ can be written as

$$
\operatorname{Br}\left(B^{-} \rightarrow e^{-} \bar{v}_{e}\right)=\tau_{B^{-}} \frac{G_{F}^{2}\left|V_{u b}\right|^{2} f_{B}^{2} M_{1}^{3}}{8 \pi}\left(\frac{m_{e}}{M_{1}}\right)^{2} \approx 10^{-11}
$$

Using the previous formula, the branching ratio for decay $B^{-} \rightarrow \mu^{+} \mu^{-} e^{-} \bar{v}_{e}$ is given by

$$
B r^{\left(B^{*}\right)}\left(B^{-} \rightarrow \mu^{+} \mu^{-} e^{-} \bar{v}_{e}\right) \approx B r\left(B^{-} \rightarrow e^{-} \bar{v}_{e}\right) \times \alpha_{e m}{ }^{2} *\left(\frac{M_{1}}{m_{e}}\right)^{2} \approx 0,5 \times 10^{-7},
$$

where the helicity suppression is reduced by emission of photon from light quark of $B^{-}$-meson.

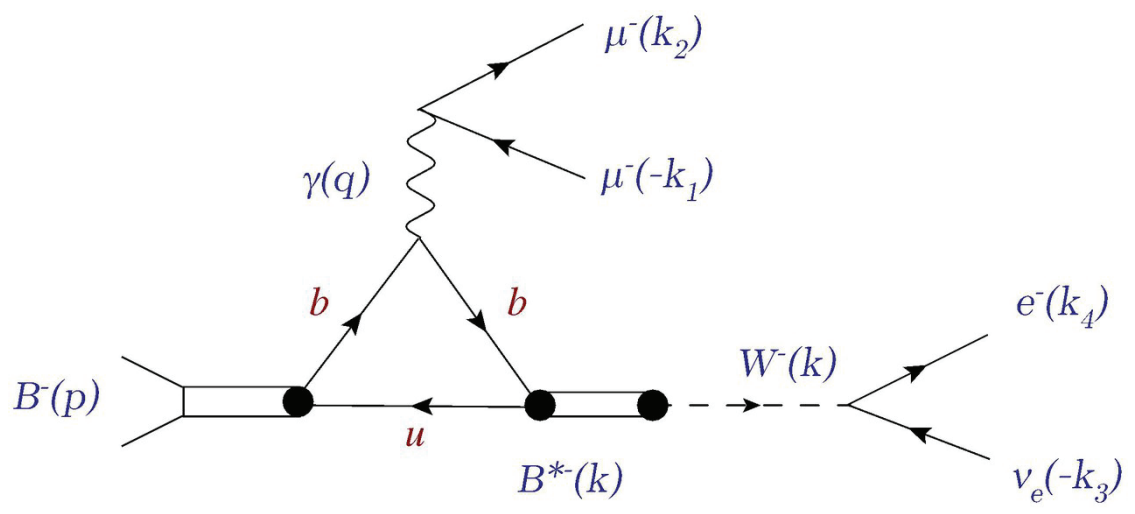

Figure 2. Emission of the virtual photon from $b$-quark in the VMD approach.

The second contribution to the amplitude describes the photon emission from the valence $b$-quark of the $B^{-}$-meson (see Fig. 2). In this case, VMD is used to calulate only $B^{*-}$ intermediate resonance contribution. The amplitude corresponding to the diagram of Fig. 2 has no singularity in the physical region of $k^{2}$, because the nearest singularity lies at $k^{2}=M_{B^{*-}}^{2}$ and $M_{B^{*-}}>M_{1}$.

In addition, the Feynman graph in Fig. 2 contains the pole in $q^{2}$-variable coming from the photon propagator. The next singularity in $q^{2}$ is at the point $q^{2}=M_{\Upsilon(1 S)}^{2}$, where $M_{\Upsilon(1 S)}$ is the mass of $\Upsilon(1 S)-$ 
meson. This pole is far beyond the kinematical decay region. Contribution of the $\Upsilon(1 S)-$ meson was included in the parametrization of transition form factor [3-5]. Therefore the part of the amplitude corresponding to the diagram of Fig. 2 can be presented as

$$
F^{(b)}\left(q^{2}, k^{2}\right)=\frac{M_{B^{*}-} f_{B^{*-}}}{q^{2}} G^{(b)}\left(q^{2}, k^{2}, M_{B^{*-}}^{2}\right) .
$$

Let us note that the diagram of Fig. 2 is suppressed compared to the diagram of Fig. 1 by the suppression factor $\lambda / m_{b}$, where $\lambda \approx 0.5 \mathrm{GeV}$ and $m_{b}$ is the b-quark mass.

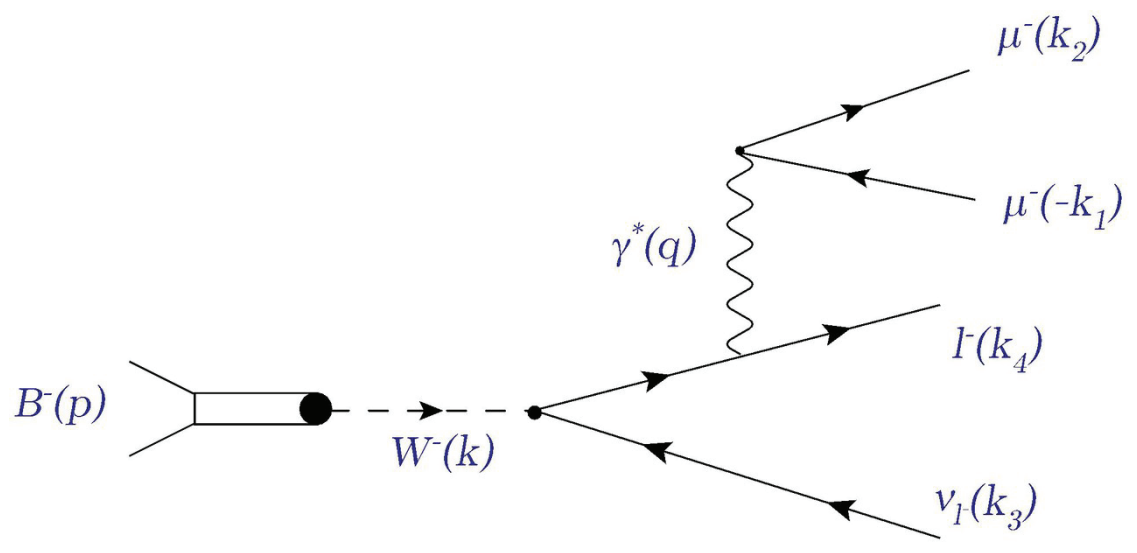

Figure 3. Bremstraulung contribution to the decay $B^{-} \rightarrow \mu^{+} \mu^{-} e^{-} \bar{v}_{e}$.

The diagram Fig. 3 corresponds to Bremsstrahlung. This diagram contains the pole from photon propagator at $q^{2}$-variable and it is significant only at small $q^{2}$-region.

Therefore, contribution of the Fig. 1 is most significant, but the other diagrams are important and have to be taken into account.

\section{Calculation of the matrix elements for the decays $B^{-} \rightarrow \ell^{+} \ell^{-} \ell^{\prime-} \bar{v}_{\ell^{\prime}}$}

Contribution to the amplitude of the decay $B^{-}(p) \rightarrow \ell^{+}\left(k_{1}\right) \ell^{-}\left(k_{2}\right) \bar{v}_{\ell^{\prime}}\left(k_{3}\right) \ell^{\prime-}\left(k_{4}\right)$ from diagram in the Fig. 1 one can calculate using the VMD model. We set $m_{\ell}=m_{\ell^{\prime}}=0$ and use the expression for the total Hamiltonian (1). For the matrix element we find

$$
\begin{aligned}
i \mathcal{M}_{f i}^{(V M D)}= & -i \frac{G_{F}}{\sqrt{2}} \frac{4 \pi \alpha_{e m}}{q^{2}} V_{u b}\left[\sum_{i=\rho^{0}, \omega} \frac{M_{2 i}^{2}}{f_{V}^{i}} \frac{1}{q^{2}-M_{2 i}^{2}+i \Gamma_{2 i} M_{2 i}} \mathcal{F}_{\mu \nu}^{(i)}(q, k)\right] \\
& \left(\bar{\ell}\left(k_{2}\right) \gamma^{v} \ell\left(-k_{1}\right)\right)\left(\overline{\ell^{\prime}}\left(k_{4}\right) \gamma^{\mu}\left(1-\gamma^{5}\right) v_{\ell^{\prime}}\left(-k_{3}\right)\right) .
\end{aligned}
$$

Taking into account the equation of motion of the lepton fields for $\mathcal{F}_{\mu \nu}^{(i)}(q, k)$ we find

$$
\mathcal{F}_{\mu \nu}^{(i)}(q, k)=\frac{2 V^{(i)}\left(k^{2}\right)}{M_{1}+M_{2 i}} \epsilon_{\mu \nu \alpha \beta} k^{\alpha} q^{\beta}-i\left(M_{1}+M_{2 i}\right) A_{1}^{(i)}\left(k^{2}\right) g_{\mu \nu}+2 i \frac{A_{2}^{(i)}\left(k^{2}\right)}{M_{1}+M_{2 i}} q_{\mu} k_{\nu} .
$$


For the diagram of Fig. 2 we have the folloving amplitude

$$
\begin{aligned}
i \mathcal{M}_{f i}^{(b)=} & -i \frac{G_{F}}{\sqrt{2}} \frac{4 \pi \alpha_{e m}}{q^{2}} V_{u b} \frac{M_{B^{*}} f_{B^{*}}}{k^{2}-M_{B^{*}}^{2}+i \Gamma_{B^{*}} M_{B^{*}}} \frac{2}{3} \frac{V_{b}\left(q^{2}\right)}{M_{1}+M_{B^{*}}} \epsilon_{\mu v p q} \\
& \left(\bar{\ell}\left(k_{2}\right) \gamma^{v} \ell\left(-k_{1}\right)\right)\left(\overline{\ell^{\prime}}\left(k_{4}\right) \gamma^{\mu}\left(1-\gamma^{5}\right) v_{\ell^{\prime}}\left(-k_{3}\right)\right),
\end{aligned}
$$

and for Fig. 3 we obtained the amplitude

$$
i \mathcal{M}_{f i}^{(\text {brem })}=-i \frac{G_{F}}{\sqrt{2}} \frac{4 \pi \alpha_{e m}}{q^{2}} V_{u b} i f_{B_{u}} g_{\mu v}\left(\bar{\ell}\left(k_{2}\right) \gamma^{\nu} \ell\left(-k_{1}\right)\right)\left(\overline{\ell^{\prime}}\left(k_{4}\right) \gamma^{\mu}\left(1-\gamma^{5}\right) v_{\ell^{\prime}}\left(-k_{3}\right)\right)
$$

\section{Numerical results for the branching ratio and differential distributions of the decay $B^{-} \rightarrow \ell^{+} \ell^{-} \ell^{\prime-} \bar{v}_{\ell^{\prime}}$}

After the calculation of the square of the matrix elemant and integration over the variables $x_{12}=q^{2} / M_{1}^{2}$ and $x_{34}=k^{2} / M_{1}^{2}$, we got the numerical prediction for the $B^{-} \rightarrow \mu^{+} \mu^{-} e^{-} \bar{v}_{e}$ branching ratio:

$$
\operatorname{Br}\left(B^{-} \rightarrow \mu^{+} \mu^{-} e^{-} \bar{v}_{e}\right) \approx 1,3 \times 10^{-7} .
$$

Let us mention that all leptons are taken massless in the calculations of the matrix element. However, the integration over $\sqrt{q^{2}}$-variable startes from double muon mass in order to avoid the singularity related to the photon propagator. Thus the integration over $x_{12}$ started from the point $x_{12}^{\min }=\left(2 m_{\mu} / M_{1}\right)^{2} \approx 0.0016$.

The double differencial distribution for the decay rates are shown in Fig. 4 and Fig. 5, where we can see peaks corresponding to the virtual photon emission and the contributions of $\rho^{0}(770)$ and $\omega(782)$ resonances.

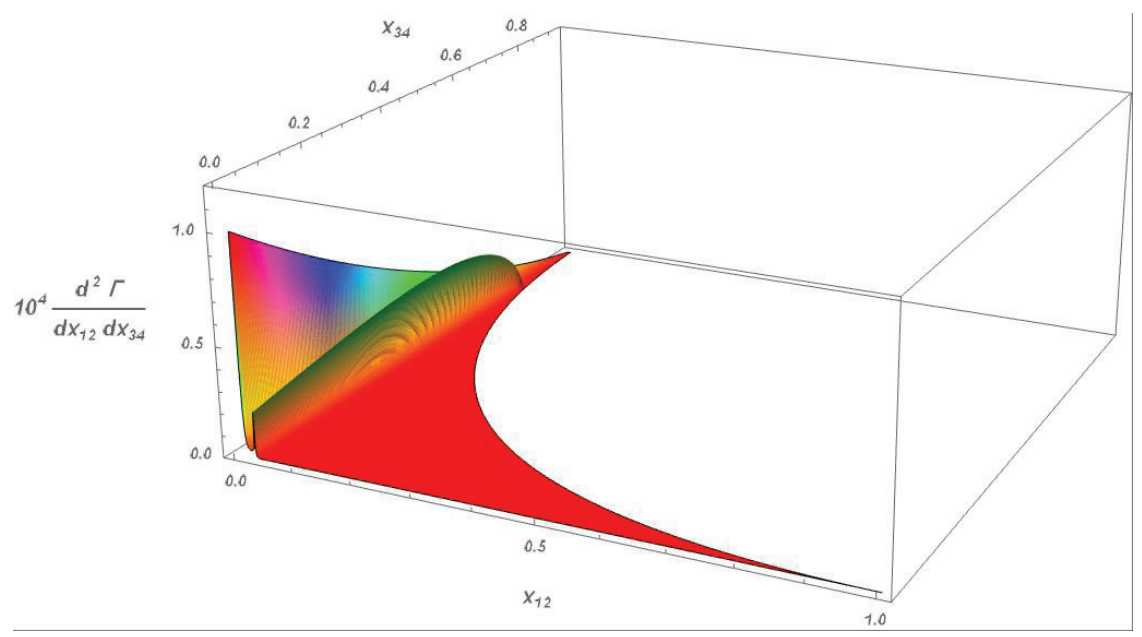

Figure 4. Double differential distributions for $\frac{d^{2} \Gamma\left(B^{-} \rightarrow \mu^{+} \mu^{-} e^{-} \bar{v}_{e}\right)}{d x_{12} d x_{34}} \times 10^{4}$.

Finally, we estimate the branching ratio of the decay of $B^{-} \rightarrow \mu^{+} \mu^{-} \mu^{-} \bar{v}_{\mu}$. Similarly to the decay $B^{-} \rightarrow \mu^{+} \mu^{-} e^{-} \bar{v}_{e}$ we take into account the contributions of the photon emission from $u$ - and $b$ - quarks 


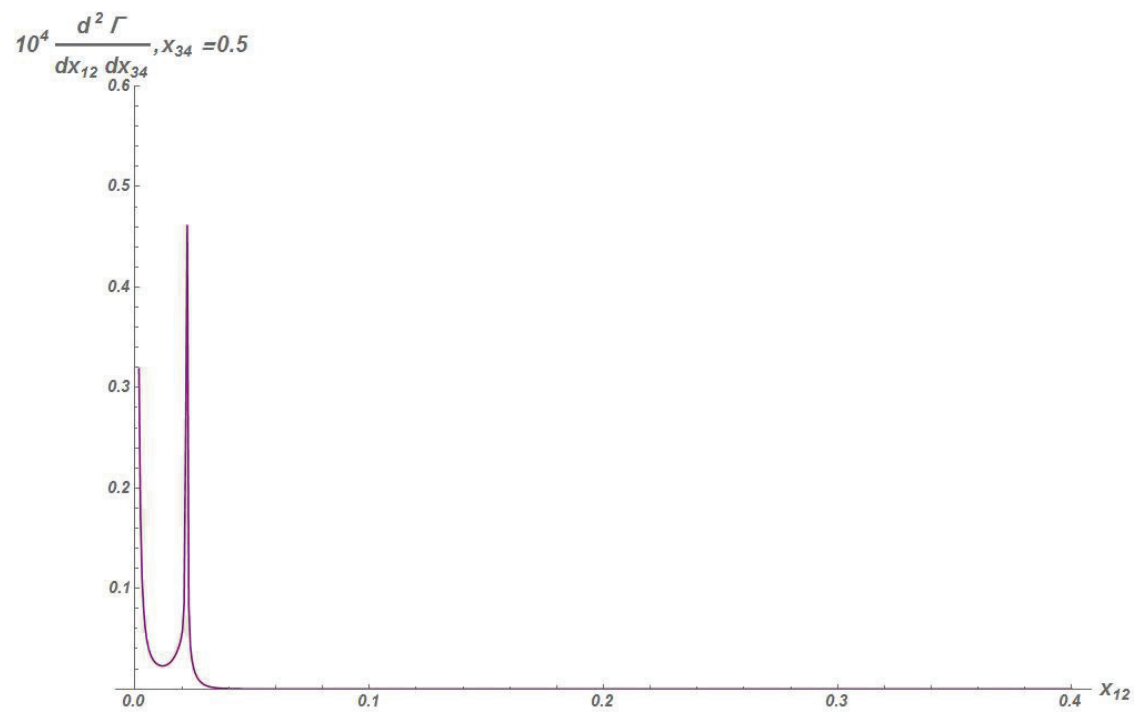

Figure 5. One-dimensional face of the double differencial distribution $\frac{d^{2} \Gamma\left(B^{-} \rightarrow \mu^{+} \mu^{-} e^{-} \bar{v}_{e}\right)}{d x_{12} d x_{34}}$ at $x_{34}=0.5$.

and Bremsstrahlung contribution. But in this case the number of diagramms doubles because of the identical fermions in the final state. So we obtain addition interferecne terms in the expression of the square matrix element.

The basic problem in estimation of branching ratio of $B^{-} \rightarrow \mu^{+} \mu^{-} \mu^{-} \bar{v}_{\mu}$ was calculation of interference part in the square matrix element. The similar processes contributed to the decay rations of $B^{-} \rightarrow \mu^{+} \mu^{-} e^{-} \bar{v}_{e}$ and $B^{-} \rightarrow \mu^{+} \mu^{-} \mu^{-} \bar{v}_{\mu}$. Therefore branching ratio for the $B^{-} \rightarrow \mu^{+} \mu^{-} \mu^{-} \bar{v}_{\mu}$ decay we can represent as

$$
\operatorname{Br}\left(B^{-} \rightarrow \mu^{+} \mu^{-} \mu \bar{v}_{\mu}\right)=\operatorname{Br}\left(B^{-} \rightarrow \mu^{+} \mu^{-} e^{-} \bar{v}_{e}\right)-B r^{(i n t e r f)}\left(B^{-} \rightarrow \mu^{+} \mu^{-} \mu^{-} \bar{v}_{\mu}\right) .
$$

Numerically $B r^{(\text {interf })}\left(B^{-} \rightarrow \mu^{+} \mu^{-} \mu^{-} \bar{v}_{\mu}\right) \approx 2.4 \times 10^{-8}$. Therefore

$$
\operatorname{Br}\left(B^{-} \rightarrow \mu^{+} \mu^{-} \mu^{-} \bar{v}_{\mu}\right) \approx 10^{-7}
$$

It means that the measurements of branching ratios for the decays $B^{-} \rightarrow \mu^{+} \mu^{-} e^{-} \bar{v}_{e}$ and $B^{-} \rightarrow$ $\mu^{+} \mu^{-} \mu^{-} \bar{v}_{\mu}$ are possible at LHCb and at Belle II.

\section{Estimations of the branching ratio for the decay $B_{s} \rightarrow \mu^{+} \mu^{-} e^{+} e^{-}$}

Using the previous result for $\operatorname{Br}\left(B^{-} \rightarrow \mu^{+} \mu^{-} e^{-} \bar{v}_{e}\right)$, we can evaluate the branching ratio of the decay $B_{s} \rightarrow \mu^{+} \mu^{-} e^{+} e^{-}$. We suppose, that nonperturbative contribution of strong interactions have the same magnitude for both decays. But the difference between these decays cosists in the diffrent contributions of weak and electromagnetic processes. This difference contanning by coefficients multiplied by the hadronic parts of the matrix elements. After calculation of this coefficient we obtain the following result:

$$
\frac{B r\left(B_{s} \rightarrow \mu^{+} \mu^{-} e^{-} e^{+}\right)}{B r\left(B^{-} \rightarrow \mu^{+} \mu^{-} e^{-} \bar{v}_{e}\right)} \approx \frac{\tau_{B_{s}}}{\tau_{B^{-}}} \frac{1}{8 \pi^{2}} \alpha_{e m}{ }^{2}\left|C_{10 A}\right|^{2} \frac{\left|V_{t b} V_{t s}^{*}\right|^{2}}{\left|V_{u b}\right|^{2}}
$$


where $\left|C_{10 A}\right|=4.64-$ the one of the Wilson coefficients for rare semileptonic $B$-decays [6]. Using the $\operatorname{Br}\left(B^{-} \rightarrow \mu^{+} \mu^{-} e^{-} \bar{v}_{e}\right)$ and numerical data from [7] gives the following estimation

$$
\operatorname{Br}\left(B_{s} \rightarrow \mu^{+} \mu^{-} e^{-} e^{+}\right) \approx 2 \times 10^{-10} .
$$

Such estimation is in good agreement with the same result given by [8], where the branching ratio of $B_{s} \rightarrow \mu^{+} \mu^{-} e^{+} e^{-}$decay was determined by taking into account only electromagnetic effects

$$
\operatorname{Br}\left(B_{s} \rightarrow \mu^{+} \mu^{-} e^{+} e^{-}\right) \approx 1.1 \times 10^{-10} .
$$

Also our result is in good agreement with theoretical estimates from $\operatorname{Br}\left(B_{s} \rightarrow \ell^{+} \ell^{-} \gamma\right)$, using the result from [9-11], we have

$$
\operatorname{Br}\left(B_{s} \rightarrow \mu^{+} \mu^{-} e^{+} e^{-}\right) \sim \alpha_{e m} \operatorname{Br}\left(B_{s} \rightarrow e^{+} e^{-} \gamma\right) \approx 1.8 \times 10^{-10} .
$$

\section{Conclusion}

We predicted the branching ratios of the decays $B^{-} \rightarrow \mu^{+} \mu^{-} e^{-} \bar{v}_{e}$ and $B^{-} \rightarrow \mu^{+} \mu^{-} \mu^{-} \bar{v}_{\mu}$. Taking into account the processes of the photon emission from $u$ - and $b$-quarks of the B-meson and the Bremsstrahlung. The following predictions are obtained:

$$
\begin{gathered}
\operatorname{Br}\left(B^{-} \rightarrow \mu^{+} \mu^{-} e^{-} \bar{v}_{e}\right) \approx 1,3 \times 10^{-7} \\
\operatorname{Br}\left(B^{-} \rightarrow \mu^{+} \mu^{-} \mu^{-} \bar{v}_{\mu}\right) \approx 10^{-7}
\end{gathered}
$$

Based on the results for the $\operatorname{Br}\left(B^{-} \rightarrow \mu^{+} \mu^{-} e^{-} \bar{v}_{e}\right)$ we get the estimation for the branching ratio of the decay $B_{s} \rightarrow \mu^{+} \mu^{-} e^{+} e^{-}$

$$
\operatorname{Br}\left(B_{s} \rightarrow \mu^{+} \mu^{-} e^{+} e^{-}\right) \sim 10^{-10} .
$$

We would like to express our deep gratitude to D. Melikhov and A. Kozachuk for multiple helpful discussions which improved the paper significantly. The work was supported by grant 16-12-10280 of the Russian Science Foundation.

\section{References}

[1] R. Aaij et al. [LHCb Collaboration], Phys. Rev. Lett. 110, 211801 (2013).

[2] R. Aaij et al. [LHCb Collaboration], JHEP 1703, 001 (2017).

[3] D. Melikhov and B. Stech, Phys. Rev. D 62, 014006 (2000).

[4] D. Melikhov, Phys. Rev. D 53, 2460 (1996).

[5] D. Melikhov, Phys. Rev. D 56, 7089 (1997).

[6] G. Buchalla, A. J. Buras and M. E. Lautenbacher, Rev. Mod. Phys. 68, 1125 (1996).

[7] C. Patrignani et al. (Particle Data Group), Chin. Phys. C 40, 100001 (2016).

[8] Y. Dincer and L. M. Sehgal, Phys. Lett. B 556, 169 (2003).

[9] F. Kruger and D. Melikhov, Phys. Rev. D 67, 034002 (2003).

[10] D. Melikhov and N. Nikitin, Phys. Rev. D 70, 114028 (2004).

[11] I. Balakireva, D. Melikhov, N. Nikitin and D. Tlisov, Phys. Rev. D 81, 054024 (2010). 